\title{
Kaninchenschnupfen - Verlauf, Therapie und Prognose
}

\author{
Barbara Glöckner
}

Kaninchenschnupfen ist eine bakterielle, hoch kontagiöse Erkrankung, deren Verlauf chronisch-rezidivierend und von verschiedenen Faktoren abhängig ist. Eine allgemeingültige Therapie gibt es nicht: Basierend auf generellen Empfehlungen muss für jedes Tier eine individuelle Behandlung eingeleitet werden. Kaninchenschnupfen ist eine typische Erkrankung der Haus- bzw. Heimtierkaninchen. Wildkaninchen fungieren nicht als Erregerreservoir.

\section{Ätiologie}

Kaninchenschnupfen wird in der Regel durch eine Mischinfektion hervorgerufen. Die Haupterreger, die auch parallel auftreten können, sind meist:

- Pasteurella multocida

- Bordetella bronchiseptica
Hinzu kommen unterschiedliche Sekundärerreger: Staphylokokken, Klebsiellen und Streptokokken sind besonders häufig nachzuweisen. Eine Beteiligung von Viren und Mykoplasmen wird diskutiert.

\section{Pathogenese}

Die Ansteckung erfolgt:

- durch direkten Kontakt von Tier zu Tier (oftmals bereits vom Muttertier auf die Jungtiere)

- indirekt über Aerosole sowie seltener über Gegenstände (z.B. unzureichend gereinigte Futternäpfe oder Tränken)

Durch die Kontagiosität unterliegen insbesondere Kaninchen in Zuchtbeständen, Tiere, die oft auf Ausstellungen oder in Tierpensionen gebracht werden, sowie alle Kaninchen aus Beständen mit regelmäßigen Zukäufen, Pflegetieren oder anderweitig bedingter häufiger Fluktuation

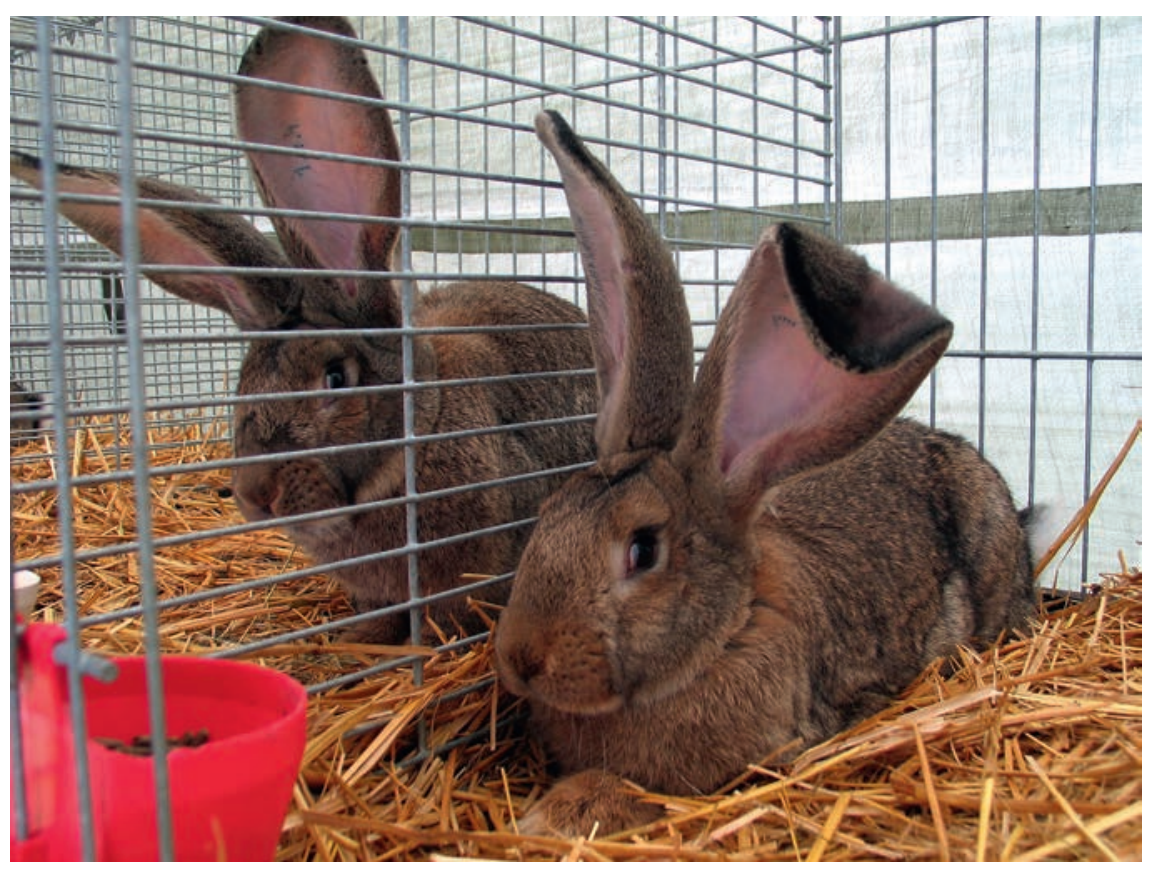

Abb. 1 Auf Ausstellungen besteht ein besonders hohes Infektionsrisiko für Kaninchenschnupfen. (c) B. Glöckner

(Tierheim, Zoofachhandel) einer hohen Infektionsgefahr ( Abb. 1).

Ob und in welchem Maße ein infiziertes Kaninchen klinisch erkrankt, ist von verschiedenen Faktoren abhängig: Im Vordergrund steht die individuelle Immunkompetenz des Einzeltieres, sodass sehr junge, alte oder durch andere Vorerkrankungen geschwächte Kaninchen besonders häufig und schwerwiegend erkranken. Hinzu kommen verschiedene Stressfaktoren, die auch bei latent infizierten adulten Tieren zu einem klinisch manifesten Kaninchenschnupfen führen können. Beispiele dafür sind:

- suboptimale Haltungsbedingungen

(viele Tiere auf zu engem Raum, mangelhafte Stallhygiene)

- Ernährungsfehler

- Umzug

- Vergesellschaftung

- Tod des Partnertieres

- Trächtigkeit und Laktation

Wetterumschwünge, feuchte Kälte oder zu trockene Heizungsluft wirken ebenfalls belastend auf die Schleimhäute des Respirationstraktes, sodass im Herbst| Winter besonders viele Neuerkrankungen und Rezidive auftreten.

\section{Klinik}

Die klinischen Symptome variieren. Sie können ein Einzeltier ebenso wie eine gesamte Kaninchengruppe betreffen und innerhalb der Gruppe in unterschiedlichem Maße ausgeprägt sein.

Klassisch ist eine reine Rhinitissymptomatik, die von gelegentlichem Niesen mit serösem Auswurf ( $\downarrow$ Abb.2) über leicht eitrigen Nasenausfluss mit nasalen Stridorgeräuschen ( $\triangleright$ Abb.3) bis zu Maulatmung aufgrund von mukopurulent verklebten Nasenöffnungen reichen kann. 


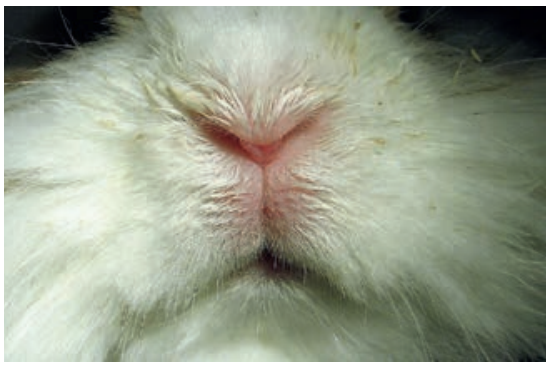

Abb. 2 Kaninchen mit milden Rhinitissymptomen und serösem Nasenausfluss. (c) B. Glöckner

Eine Verminderung der Futteraufnahme bis zur Inappetenz kann aufgrund der Einschränkungen des Geruchs- und Geschmackssinns auftreten. Weichen Kaninchen aufgrund verlegter Nasenöffnungen auf Maulatmung aus, stellen sie ebenfalls die Futteraufnahme ein, da der Kauvorgang die Atmung weiter behindern würde.

Zahlreiche Verlaufsformen betreffen auch den Tränennasenkanal und die Konjunktiven, sodass bei eitrigen Dakryozystitiden und Konjunktivitiden ( $\triangleright$ Abb.4) stets ein Kaninchenschnupfen differentialdiagnostisch in Erwägung gezogen werden muss.

Über eine Erregerstreuung durch die Eustachische Röhre kann zudem eine Otitis media oder interna ein- oder beidseits entstehen. Betroffene Tiere zeigen Einschränkungen des Gehörs, Gleichgewichtsstörungen, gelegentlich Nystagmus und bei einseitiger Erkrankung eine Kopfschiefhaltung.

Breiten sich Erreger in den unteren Respirationstrakt aus, so ist die Entstehung z.B. einer Tracheitis, Bronchitis oder Bronchopneumonie möglich. Bei ungünstigstem Verlauf bilden sich Abszesse in der Lunge. Dyspnoe und Apathie sowie wiederum Inappetenz gehören dann zusätzlich zu den Symptomen.

Durch eine Erregerstreuung können auch vom Respirationstrakt unabhängig weitere Veränderungen auftreten. Folgendes lässt sich häufig mit Erregern des Kaninchenschnupfens in Zusammenhang bringen:

- Abszesse oder Entzündungen in der Leber, in Lymphknoten oder im Rü-

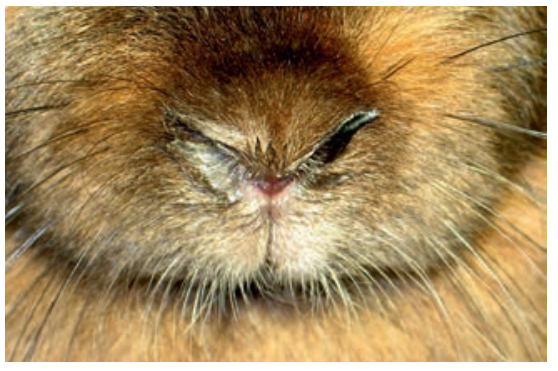

Abb. 3 Kaninchen mit mäßigem eitrigen Nasenausfluss. (C) B. Glöckner

ckenmark (insbesondere aufgrund von Absiedlungen von Pasteurellen)

- lokalisierte oder generalisierte Arthritiden

- Entzündungen der Gebärmutter (beim Kaninchen ansonsten eher selten)

- Uveitiden

Vorwiegend bei geschwächten oder sehr jungen Tieren können sich durch eine Erregerabsiedlung eine generalisierte Pasteurellose und damit auch eine Septikämie entwickeln.

\section{Diagnose}

Die Anamnese und das klinische Bild können bereits eine Verdachtsdiagnose nahelegen. Wird ein Kaninchen mit Nasen- und ggf. Augenausfluss sowie Sekretspuren an den Innenseiten der Vorderläufe, die durch das Putzverhalten entstehen, vorgestellt, ist stets eine Kaninchenschnupfenerkrankung abzuklären.

\section{Klinische Untersuchung}

Im Verlauf der klinischen Allgemeinuntersuchung sollte ein Hauptaugenmerk auf die Auskultation gelegt werden. Diese ist häufig durch den lauten nasalen Stridor erschwert und muss daher ausreichend lange und gründlich durchgeführt werden. Bei der Untersuchung der äußeren Gehörgänge ist auf Vorwölbungen des Trommelfells zu achten, die auf eine Entzündung im Mittelohrbereich hinweisen könnten. Im Zuge der Augenuntersuchung ist es insbesondere bei zunächst unauffälligem Befund sinnvoll, das untere Augenlid leicht nach unten zu ziehen und durch vorsichtige Massage rostral des Auges zu überprüfen, ob sich Sekret aus dem Tränennasenkanal entleeren lässt.

\section{Weiterführende Untersuchungen}

Das Anfertigen von Röntgenaufnahmen des Thorax ist immer sinnvoll bei:

- bronchialen Atemgeräuschen

- punktueller Dämpfung der Herztöne, die durch einen Lungenabszess verursacht werden kann

Bei akuter Erkrankung sind im Blutbild in der Regel deutliche Leukozytosen festzustellen. Auch bei chronischen Verlaufsformen können meist noch Verschiebungen des Differentialblutbildes (Neutrophilie bei gleichzeitiger Lymphopenie) beobachtet werden.

Eine bakteriologische Untersuchung des Nasenausflusses gehört nicht zur Routinediagnostik, da die Erreger nur unregelmäßig nachgewiesen werden können. Insbesondere bei Abstrichen aus dem Bereich der Nasenöffnungen sind falsch negative Befunde häufig. Außerdem werden oftmals ubiquitär vorkommende Keime aus dem Fell oder der äußeren Haut oder aber Sekundärerreger nachgewiesen, sodass die Befunde stets mit Vorsicht zu interpretieren sind. Auch Nasenspülproben erbringen häufig kein sicher interpretierbares Resultat.

Die bakteriologische Untersuchung des Nasenausflusses stellt kein zuverlässiges diagnostisches Verfahren dar. Falsch negative Ergebnisse sowie die Isolierung ubiquitärer Bakterien und Sekundärerreger kommen häufig vor.

Eine aus der Tiefe der Nasenhöhle entnommene Tupferprobe liefert in der Regel zuverlässigere Ergebnisse. Hierzu

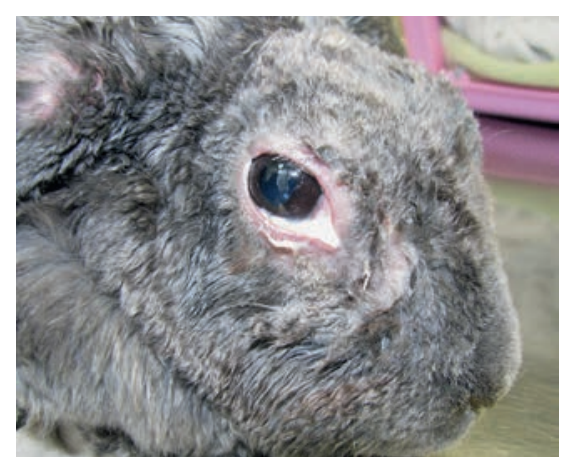

Abb. 4 Dakryozystitiden treten häufig in Verbindung mit Kaninchenschnupfen auf. (c) B. Glöckner 
ist jedoch eine kurze Sedation notwendig, deren Risiko je nach Zustand des Patienten sorgfältig abgewogen werden muss. Mit einem möglichst dünnen Abstrichbesteck, wie es z.B. für Konjunktivalabstriche eingesetzt wird, kann dann Sekret entnommen werden, in dem sich oftmals noch vermehrungsfähige Keime befinden. Auch hier ist es jedoch möglich, einen falsch negativen Befund oder lediglich den Nachweis von Sekundärerregern zu erhalten: Dies ist bei der Interpretation zu berücksichtigen.

\section{Differentialdiagnosen}

Sowohl für die Schnupfensymptomatik als auch für die Symptome der unteren Atemwege kommen differentialdiagnostisch akute bakterielle Infektionen anderer Genese infrage.

Insbesondere bei eitrigem Augen- und Nasenausfluss ist eine Dakryozystitis abzuklären, die sowohl ein- als auch beidseits auftreten kann. Die möglichen Ursachen sind:

- Verlegung oder Perforation des Tränennasenkanals aufgrund apikalen Zahnwachstums

- akute bakterielle Infektionen

- Reizungen nach Zugluft

Apikale Granulome oder Abszedierungen können zudem zu oronasalen Fisteln führen. Durch den Einbruch in die Nasenhöhle tritt meist einseitig eitriger Ausfluss auf.

Ebenfalls überwiegend einseitig sind Veränderungen zu beobachten, die durch einen Fremdkörper bedingt sind. Oftmals sind Heu- oder Grashalme ursächlich, die entweder direkt in die Nasenöffnung oder retrograd über den Rachenraum in die Nasenhöhle gelangt sind.

Liegt lediglich seröser Nasen- und ggf. Augenausfluss vor, so ist auch ein allergisches Geschehen zu berücksichtigen.

Im Falle einer Kopfschiefhaltung ist stets an eine Encephalitozoonose zu denken, die auch parallel bei einem durch chronischen Kaninchenschnupfen immunsupprimierten Tier auftreten kann. Zudem ist eine Otitis durch Milben (Psoroptes cuniculi) abzuklären.
Eine Erregerstreuung oder eine generalisierte Pasteurellose kann sehr vielfältige Veränderungen verursachen: In diesen Fällen sind unterschiedliche Differentialdiagnosen abzuklären.

\section{Therapie}

Es gibt keine Therapie, die immer und bei jedem Kaninchenschnupfen-Patienten die optimale Behandlung darstellt. Zum einen handelt es sich um eine Mischinfektion: Pasteurellen und/oder Bordetellen liegen in den meisten Fällen der Erkrankung zugrunde, aber die jeweiligen Sekundärerreger und damit auch die Resistenzen variieren. Zum anderen spielen zahlreiche äußere Faktoren (z. B. Haltungs- und Fütterungsbedingungen sowie die individuelle Immunkompetenz) eine Rolle. Daher müssen die grundsätzlichen Therapieempfehlungen ( $\vee$ Tab. 1) für den jeweiligen Bestand bzw. Patienten individuell zusammengestellt werden.

Zunächst ist jedoch mit dem Besitzer zu besprechen, dass es sich um eine chronisch-rezidivierende Erkrankung handelt.

Eine vollständige Ausheilung kann in der Regel nicht erreicht werden, jedoch sind oftmals lange symptomfreie Intervalle bzw. lange Zeiträume mit gutem Allgemeinbefinden zu erwarten, wenn das Kaninchen auf die Therapie gut anspricht.

Kaninchen mit eitrigem Nasenausfluss erhalten über einen Zeitraum von mindestens 10 Tagen ein Antibiotikum. Hier haben sich Fluorchinolone als Wirkstoffe der 1. Wahl bewährt, die auch für eine langfristige Gabe geeignet sind. Vorteilhaft sind neben der vielfach nachgewiesenen guten Wirksamkeit und Verträglichkeit zudem die nur 1-mal tägliche Applikation sowie ein vergleichsweise geringes Applikationsvolumen bei oraler Verabreichung, was bei Tieren mit Dyspnoe ein nicht zu unterschätzender Faktor ist.

Erscheinen Fluorchinolone in hochgradigen Erkrankungsfällen allein nicht ausreichend wirksam, so können sie z.B. mit einem Langzeit-Cephalosporin, das aus- schließlich parenteral verabreicht werden darf, kombiniert werden (z.B. Enrofloxacin und Cefovecin). Das Cephalosporin kann auch als Einzelwirkstoff parenteral eingesetzt werden, wenn die Antibiotika der 1. Wahl keine Wirksamkeit zeigen. Dabei ist zu beachten, dass die Wirkdauer des Depot-Präparats deutlich kürzer ist, als für Hund und Katze angegeben.

Als Alternative ist in hartnäckigen Fällen der Einsatz von Penicillinen parenteral unter Unterstützung der Darmflora möglich. Liegt ein Antibiogramm vor, so ist dieses vorrangig zu berücksichtigen.

Außer bei akuten Verschlechterungen oder bei Unverträglichkeitsanzeichen sollte ein Antibiotikum stets mindestens 5 Tage verabreicht werden, bevor die Therapie umgestellt wird.

Zusätzlich ist ein Mukolytikum zu verordnen (z.B. Bromhexin oder Acetylcystein). Viele Kaninchen sprechen zudem sehr gut auf die Gabe eines Paramunitätsinducers an, der entweder parallel zur antibiotischen Therapie oder in leichteren Erkrankungsfällen auch als alleinige Unterstützung kurweise verabreicht werden kann. Der positive Effekt nach einer Kur kann oftmals durch eine weitere längerfristige Verabreichung im Abstand von 1-2 Wochen aufrechterhalten werden.

Sehr hilfreich sind auch Inhalationen, die der Besitzer zu Hause durchführen kann. Als besonders vorteilhaft haben sich hierbei Inhalationsgeräte aus der Kleinkindmedizin erwiesen (z.B. Modelle des PariBoy $\left.{ }^{\circledR}\right)$, mit denen entweder eine reine $\mathrm{NaCl}$-Lösung vernebelt werden oder auch ein Mukolytikum zugesetzt werden kann. Die Inhalationsdauer sollte 10 Minuten nicht überschreiten. Gleiches gilt auch für Warminhalationen, bei denen beispielsweise die mukolytische Wirkung eines Thymianaufgusses genutzt wird. Sowohl Warm- als auch Kaltinhalationen werden meist unproblematisch toleriert und können besonders leicht in einer abgedeckten Transportbox durchgeführt werden, vor die das Inhalat gestellt bzw. an der der Inhalator befestigt wird. 
Tab. 1 Kaninchenschnupfen: allgemeine Therapieempfehlungen.

\begin{tabular}{|c|c|c|c|}
\hline Wirkstoff & Präparat & Dosierung & $\begin{array}{l}\text { Für Kaninchen } \\
\text { zugelassen? }\end{array}$ \\
\hline \multicolumn{4}{|l|}{ Antibiotikum } \\
\hline Enrofloxacin & z. B. Baytril ${ }^{\circledR}$, Bayer Vital GmbH & $10 \mathrm{mg} / \mathrm{kg} 1 \times$ tgl. s.c.oder p.o. & $\mathrm{Ja}$ \\
\hline Marbofloxacin & z. B. Marbocyl ${ }^{\circledR}$, Vétoquinol GmbH & $4 \mathrm{mg} / \mathrm{kg} 1 \times \mathrm{tgl}$. s.c.oder p.o. & Nein \\
\hline Pradofloxacin & Veraflox ${ }^{\circledR}$, Bayer Vital GmbH & 7,5 mg/kg $1 \times$ tgl. p.o. & Nein \\
\hline Cefovecin & Convenia $^{\circledR}$, Zoetis Deutschland $\mathrm{GmbH}$ & $8 \mathrm{mg} / \mathrm{kg} 1 \times$ wöchentlich s.c. & Nein \\
\hline \multicolumn{4}{|l|}{ Mukolytikum } \\
\hline Bromhexin & $\begin{array}{l}\text { Bisolvon }{ }^{\circledR} \text {, Boehringer Ingelheim Pharma GmbH \& Co. KG } \\
\text { (Injektionslösung) }\end{array}$ & $0,5 \mathrm{mg} / \mathrm{kg} 2 \times$ tgl. p.o. & Nein \\
\hline Acetylcystein & z.B. NAC-ratiopharm ${ }^{\circledR}$, Ratiopharm GmbH (Injektionslösung) & $3 \mathrm{mg} / \mathrm{kg} 2 \times$ tgl. p.o. & Nein \\
\hline \multicolumn{4}{|c|}{ Paramunitätsinducer } \\
\hline Parapoxvirus ovis & Zylexis $^{\circledR}$, Zoetis Deutschland $\mathrm{GmbH}$ & $\begin{array}{l}\text { kurweise am 1., 3. und } 8 \text {. Tag je } 1 \mathrm{ml} \\
\text { s.c., ggf. weiter im Abstand von } \\
\text { 1-2 Wochen }\end{array}$ & Nein \\
\hline \multicolumn{4}{|c|}{ Flankierende Maßnahmen } \\
\hline $\begin{array}{l}\text { Spülungen des } \\
\text { Tränennasenkanals }\end{array}$ & $\begin{array}{l}\text { Sterile NaCl-Lösung, letzter Spülgang mit Floxal }{ }^{\circledR}, \text { Zylexis }^{\circledR} \text {, } \\
\text { oder Mucosa compositum ad us. vet. }\end{array}$ & bedarfsweise & \\
\hline Inhalationen & $\begin{array}{l}\text { NaCl-Lösung (ggf. + Mukolytikum) } \\
\text { Thymianaufguss (warm) }\end{array}$ & $1 \times$ tgl., max. 10 Minuten & \\
\hline
\end{tabular}

Sind die Nasenöffnungen des Patienten mit Sekret verklebt, so sollten sie regelmäßig mit lauwarmem Wasser gereinigt werden. Größere Sekretmengen können ggf. mit einer Spritze abgesaugt oder vorsichtig ausgespült werden. Hierbei sollte eine feine, weiche Ernährungssonde oder eine flexible Zitzenkanüle aus Kunststoff aus der Großtiermedizin aufgesetzt werden. Auch Meerwasser-Nasentropfen für Kleinkinder können Linderung bringen.

Zur weiteren Unterstützung des Immunsystems und der Regeneration der Schleimhäute haben sich verschiedene homöopathische Komplexmittel sehr bewährt ( Tab.2). Zu homöopathischen Monopräparaten können hier keine pauschalen Empfehlungen ausgesprochen werden, denn sie sind aufgrund der jeweiligen Anamnese und des jeweiligen Erkrankungsverlaufes individuell zusammenzustellen.

Bei Kaninchen mit Dakryozystitiden muss der Tränennasenkanal gespült werden. Dies kann mit steriler Kochsalzlösung erfolgen, wobei es sich bewährt hat, mit dem letzten „Spülgang“ antibiotische Augentropfen (z.B. Floxal ${ }^{\circledR}$, Bausch \& Lomb), immunstimulierende Präparate (z.B. Zylexis ${ }^{\circledR}$, Zoetis Deutschland GmbH) oder Lösungen, die die Regeneration der Schleimhaut fördern (z.B. Mucosa compositum ad us. vet., Heel $\mathrm{GmbH}$ ) zu instillieren.

Muss aufgrund einer Konjunktivitis eine lokale Medikation am Auge erfolgen, so sind auch hier stets wässrige Augentropfen (z.B. Floxal ${ }^{\circledR}$, Bausch \& Lomb; Poly$\operatorname{spectran}^{\circledR}$, Alcon Pharma GmbH) oder alternativ gelförmige Substanzen einzusetzen. Augensalben haben zum einen den Nachteil, dass viele Kaninchen überempfindlich auf die Salbengrundlage mit Rötungen und Schwellungen der Lider reagieren, zum anderen verkleben sie aufgrund ihrer Textur oftmals den Tränenpunkt.

Inappetente Kaninchen müssen mit einer bedarfsgerechten Nahrung zugefüttert werden (z.B. Critical Care, Albrecht GmbH; RodiCare $^{\circledR}$ instant, alfavet Tierarzneimittel GmbH; Herbi Care Plus, WDT).
Parallel zur Therapieeinleitung sollten die Haltungs- und Fütterungsbedingungen optimiert werden. Zudem ist auf eine hohe Flüssigkeitsaufnahme aller erkrankten Tiere zu achten: Frisches Wasser sollte stets bereitgestellt werden. Bei inappetenten Kaninchen kann die Flüssigkeitszufuhr über die Konsistenz des angerührten Nahrungsbreis geregelt werden, im Bedarfsfall kann auch zusätzlich Wasser oder Tee per Spritze verabreicht werden. Bei der Allgemeinuntersuchung ist der Hautturgor als Parameter für den Hydratationsgrad zu überprüfen.

Sind aufgrund von Erregerabsiedlungen weitere Veränderungen aufzufinden, so ist die Behandlung entsprechend anzupassen.

\section{Prognose}

Die Prognose ist meist abhängig vom $\mathrm{Zu}$ stand der Tiere bei der Erstvorstellung, eine vollständige Ausheilung ist in der Regel nicht zu erwarten. Sind erstmals Symptome aufgetreten und beschränken 
sich diese auf den oberen Atmungstrakt, so ist die Prognose günstig einzuschätzen und bei einem Großteil der Patienten sind längere symptomfreie Intervalle gelegentlich auch eine dauerhafte Symptomfreiheit - zu erwarten.

Auch dauerhaft asymptomatische Kaninchen dienen weiter als Erregerreservoir und damit als Infektionsquelle.

Ein Teil der Patienten zeigt ständig Erkrankungsanzeichen wie vermehrtes Niesen und Nasenausfluss. Diese Symptome sind häufig jedoch gut durch regelmäßige Unterstützung des Immunsystems, bedarfsweise Gaben von Mukolytika bzw. bedarfsweise Inhalationen in einem Rahmen zu halten, der das Allgemeinbefinden nicht einschränkt.

Bestehen die Erkrankungsanzeichen bereits über längere Zeit oder sind schon Veränderungen von Bronchien und Lunge festzustellen, ist die Prognose vorsichtig zu stellen. Bei septikämischem Verlauf ist die Prognose äußerst ungünstig; liegen Organabszesse vor, so ist sie kurz- bis mittelfristig infaust.

\section{Prophylaxe}

\section{Heimtierhaltung}

Für Heimtierkaninchen zählt die Impfung gegen Kaninchenschnupfen nicht zu den Core-Impfungen. Gesunde Kaninchen in einer Heimtierhaltung haben aufgrund der Übertragungswege nur ein äußerst geringes Risiko, sich mit dem Kaninchenschnupfen-Komplex zu infizieren und daran zu erkranken. Lediglich Zukäufe und Aufenthalte in Pensionen bergen ein Risiko, das auch durch eine Quarantäne nicht sicher ausgeschlossen werden kann. Sind Kaninchen bereits bei Zukauf infiziert, so kann auch eine Impfung die Manifestation der Erkrankung nicht sicher verhindern, sondern in einigen Fällen sogar forcieren. In der Heimtierhaltung mit kleinen Bestandsgrößen sind daher optimale Haltungs- und Fütterungsbedingungen als wichtigste Prophylaxemaßnahmen anzusehen.

Tab. 2 Kaninchenschnupfen: unterstützende homöopathische Komplexmittel.

\begin{tabular}{|c|c|c|}
\hline Homöopatisches Komplexmittel & Dosierung & $\begin{array}{l}\text { Für Kaninchen } \\
\text { zugelassen? }\end{array}$ \\
\hline \multicolumn{3}{|l|}{ Unterstützung des Immunsystems } \\
\hline Echinacea compositum ad us. vet., Heel GmbH & $\begin{array}{l}0,5 \text { ml } 1 \times \text { tgl. p.o. } \\
\text { oder s.c. }\end{array}$ & Nein \\
\hline Engystol ${ }^{\circledR}$ ad us. vet., Heel GmbH & $\begin{array}{l}0,5 \text { ml } 1 \times \text { tgl. p.o. } \\
\text { oder s.c. }\end{array}$ & Nein \\
\hline Pet-M ${ }^{\circledR}$, PlantaVet GmbH & $\begin{array}{l}1 \text { ml } 1 \text { × tgl. p.o. } \\
\text { oder s.c. }\end{array}$ & Ja \\
\hline \multicolumn{3}{|l|}{ Mukolyse } \\
\hline Membrana nasalium comp. PLV, PlantaVet GmbH & $\begin{array}{l}0,5 \text { ml } 1 \times \text { tgl. p.o. } \\
\text { oder s.c. }\end{array}$ & Ja \\
\hline Sinupret $^{\circledR}$, Bionorica & $\begin{array}{l}\text { z. B. als Liquitabs: } \\
1 \text { Tbl. } 1 \times \text { tgl. p.o. }\end{array}$ & Nein \\
\hline \multicolumn{3}{|l|}{ Regeneration der Schleimhaut } \\
\hline Mucosa compositum ad us. vet., Heel GmbH & $\begin{array}{l}0,5 \text { ml } 1 \times \text { tgl. p.o. } \\
\text { oder s.c. }\end{array}$ & Nein \\
\hline \multicolumn{3}{|l|}{ Unterstützung bei akuter und chronischer Rhinitis } \\
\hline Euphorbium compositum ad us. vet., Heel GmbH & $\begin{array}{l}0,5 \text { ml } 1 \times \text { tgl. p.o. } \\
\text { oder s.c. }\end{array}$ & Nein \\
\hline \multicolumn{3}{|c|}{ Unterstützung bei akuter Rhinitis, Konjunktivitis und Dakryozystitis } \\
\hline Euphrasia Injeel ${ }^{\circledR}$, Heel GmbH & $\begin{array}{l}0,5 \text { ml } 1 \times \text { tgl. p.o. } \\
\text { oder s.c. }\end{array}$ & Nein \\
\hline Euphrasia-logoplex ${ }^{\circledR}$ Globuli, Ziegler GmbH & $\begin{array}{l}2-5 \text { Globuli } \\
2-3 \times \text { tgl. p.o. }\end{array}$ & Ja* \\
\hline
\end{tabular}

\section{Zucht- und größere Liebhaber- bestände, Tierheime}

Eine Impfung gegen Kaninchenschnupfen kommt insbesondere für Zuchtbestände und Tiere, die regelmäßig auf Ausstellungen gebracht werden, infrage. Auch in Liebhaberbeständen mit häufigen Zukäufen und Vermittlungen oder in Tierheimen kann eine Impfung sinnvoll sein, um den Infektionsdruck zu senken. Ein vollständiger Schutz gegen die Erreger des Kaninchenschnupfenkomplexes ist jedoch nicht zu erwarten, da der Impfstoff nur einen Teil abdecken kann (Pasteurella multocida Serovar A, Pasteurellamultocida-Toxin des Serovars D sowie Bordetella bronchiseptica).

Eine Impfung erfolgt frühestens am 28. Lebenstag, die Grundimmunisierung wird mit einer Boosterung nach 14 Tagen abgeschlossen. Eine regelmäßige, halbjährliche Wiederholungsimpfung ist nötig, um den Impfschutz aufrechtzuerhalten. Auch hier sind optimale Haltungs- und Fütterungsbedingungen wiederum als weitere unverzichtbare Bausteine der Prophylaxe anzusehen.

Online zu finden unter

http://dx.doi.org/10.1055/s-0033-1361567

Literatur

Literatur bei der Autorin

\section{Dr. Barbara Glöckner}

c/o Tierarztpraxis Dr. H. Brieger

Anhaltiner Str. 2A

14163 Berlin 